\title{
FREQUENCY OF CELLS EXPRESSING CD44, A HEAD AND NECK CANCER STEM CELL MARKER: CORRELATION WITH TUMOR AGGRESSIVENESS
}

\author{
Benzion Joshua, MD, ${ }^{1}$ Michael J. Kaplan, MD, ${ }^{1}$ Ilana Doweck, MD, ${ }^{2}$ Reetesh Pai, MD, ${ }^{3}$ \\ Irving L. Weissman, MD, ${ }^{4}$ Mark E. Prince, MD, ${ }^{5}$ Laurie E. Ailles, $\mathrm{PhD}^{6}$ \\ ${ }^{1}$ Department of Otolaryngology-Head and Neck Surgery, Stanford University Medical Center, Stanford, California. E-mail: \\ benbat7@yahoo.com \\ ${ }^{2}$ Departments of Otolaryngology-Head and Neck Surgery, Carmel Medical Center, Haifa, Israel \\ ${ }^{3}$ Department of Pathology, Stanford University Medical Center, Stanford, California \\ ${ }^{4}$ Department of Developmental Biology, Stanford Institute of Stem Cell Biology and Regenerative Medicine, Stanford \\ University School of Medicine, Stanford, California \\ ${ }^{5}$ Department of Otorhinolaryngology-Head and Neck Surgery, University of Michigan, Ann Arbor \\ ${ }^{6}$ Ontario Cancer Institute, Campbell Family Cancer Research Institute, and Department of Medical Biophysics, University \\ of Toronto, Toronto, Ontario, Canada
}

Accepted 22 October 2010

Published online 14 February 2011 in Wiley Online Library (wileyonlinelibrary.com). DOI: 10.1002 /hed.21699

\begin{abstract}
Background. We previously identified by flow cytometry a Lineage-CD44+ (Lin-CD44+) subpopulation of cells with cancer stem cell properties in head and neck squamous cell carcinoma (HNSCC). We now correlate clinical and histologic factors with Lin-CD44+ cell frequency.

Methods. The study included 31 patients with HNSCC, of whom $87 \%$ had stage IV disease. The frequency of Lin-CD44+ cells and the success of xenografting patient tumors in mice were correlated with clinical and pathologic data.

Results. The mean frequency of Lin-CD44+ cells was 25\% $(0.4 \%-81 \%)$. It was $36 \%$ in patients who had recurrence versus $15 \%$ for those without recurrence $(p=.04)$. Successful xenograft implantation occurred in $53 \%$. Seventy-five percent of patients with successful xenografts had recurrence versus $21 \%$ of patients with unsuccessful xenografts $(p=.003)$.

Conclusions. Successful xenograft implantation and a high frequency of Lin-CD44+ cells correlate with known poor prognostic factors such as advanced T classification and recurrence. These findings may support the stem cell concept in HNSCC. () 2011 Wiley Periodicals, Inc. Head Neck 34: 42-49, 2012
\end{abstract}

Keywords: head and neck; squamous cell carcinoma; cancer; stem cells; CD44

Head and neck squamous cell carcinoma (HNSCC) affects more than 40,000 new patients in the United States each year. ${ }^{1}$ Advances in treatment have improved quality of life, and there have been improvements in survival associated with nasopharyngeal carcinoma $^{2}$ and human papillomavirus (HPV)-associated oropharyngeal carcinomas. ${ }^{3,4}$ However, for the typical HNSCCs associated with tobacco and alcohol use, sur-

Correspondence to: B. Joshua

(C) 2011 Wiley Periodicals, Inc vival rates have changed little in the last 40 years. $^{4}$ The mortality rate remains high because of advanced and recurrent locoregional disease, as well as distant metastases. Better understanding of the biology of HNSCC is required to define relevant targets and to develop novel therapeutic approaches.

Stem Cells in Cancer. Stratified squamous epithelium contains a basal layer of cells, some or all of which are the source of epithelial replenishment during the normal life cycle of the tissue and in response to injury. In other words, normal stem cells in the basal layer are the functional reservoir for the normal homeostasis within a correctly regulated squamous cell microenvironmental niche. Such cells generate daughter cells on division, with some remaining as a stem cell (selfrenewing) and some morphologically and functionally maturing as they begin their migration toward the surface and die. This migration and regulated response to environmental signals from their niche is normal, with morphologic and functional heterogeneity.

Epithelial tumors such as HNSCC contain both morphologic and functional cellular heterogeneity, ${ }^{5,6}$ as would be expected if they arise from dysregulated stem or progenitor cells as opposed to the simple clonal expansion of a mutated cell. This observation supports the cancer stem cell hypothesis for HNSCC: normal stem or progenitor cells become dysregulated, leading to a cancer stem cell, with the tumor hierarchically organized. Some cells retain the self-renewal capacity inherent in the normal stem cell, and these cancer stem cells drive tumorigenesis through self-renewal and differentiation. The cancer stem cells also give rise to a large population of differentiated progeny that make up the bulk of tumor 
but lack the tumorigenic potential of the stem cell, just as the cells above the basal layer of the stratified epithelium are destined to die as they migrate to the surface. In support of this hypothesis, recent studies have shown that, similar to leukemia and other hematologic malignancies, ${ }^{7-11}$ tumorigenic and nontumorigenic populations of cancer cells can be isolated from a variety of tumor types on the basis of their expression of cell-surface markers. ${ }^{12,13}$ Although not all accept this hypothesis, ${ }^{14}$ support of the cancer stem cell hypothesis in a variety of solid tumor types remains strong. ${ }^{15,16}$

Numerous prognostic markers exist for HNSCC, with clinical and histologic factors remaining the most commonly used. Reliable correlates of the likelihood of recurrence and poorer survival in HNSCC remain the mainstay of the TNM system: extent of the tumor and the presence of neck metastases. ${ }^{17}$ Histologic findings, such as extracapsular extension, perineural invasion, and lymphovascular invasion are also correlated with worse prognosis. Various immunohistochemical markers have been studied as well. ${ }^{18}$

Recently it was shown ${ }^{19}$ that a gene signature in breast cancer stem cells identified tumors with a worse prognosis. The ability to differentiate a stem cell gene signature in unfractionated tumor tissue from patients with a poor prognosis suggests that these tumors contain a higher frequency of cancer stem cells.

Applying methods successfully used to identify cancer stem cells in breast cancer and other solid tumors, HNSCC tumors were previously shown to contain a distinct population of cancer cells defined by their lack of expression of markers associated with non-tumor cell lineages present within the tumors (hematopoietic cells, endothelial cells, and fibroblasts, referred to as Lin+ cells) and expression of CD44 (thus referred to as "Lin-CD44+ cells"). These cells had the ability to produce tumors in immunocompromised mice, whereas the Lin-CD44- population from the same patient samples did not have the ability to initiate tumors in mice. Tumors initiated by the Lin-CD44+ population recapitulated the original tumor heterogeneity, and serial transplantation indicated self-renewal ability when xenotransplanted into mice. ${ }^{20,21}$ Thus the Lin-CD44+ cells in HNSCC possess properties classically attributed to stem cells. The importance of CD44 as a tumor-initiating cell marker was also shown in studies with cell lines rather than fresh human tissue. ${ }^{22,23}$

During the course of analyzing many HNSCCs, it was observed that these tumors have a broad range of Lin-CD44+ cell frequencies. Another observation was that approximately $50 \%$ of tumors were successfully xenografted into mice, even when implanting large numbers of cells or tissue fragments. It was postulated that HNSCC rich in Lin-CD44+ cells would correlate with a worse prognosis because of a higher frequency of stem cells, similar to the findings of the breast cancer study. ${ }^{19}$ It was also suggested that tumors that could be successfully xenografted in mice may represent a more aggressive phenotype.

\section{MATERIALS AND METHODS}

Between February 2004, and November 2006, fresh tumors were obtained from the resected tissue of patients undergoing surgery for advanced head and neck squamous cell carcinoma at Stanford University Medical Center. The use of human tissues and animal experiments were performed in accordance with procedures and guidelines established by the Stanford University Institutional Review Board and Administrative Panels for Laboratory Animal Care.

As reported earlier, ${ }^{20}$ a mouse xenograft model of HNSCC was developed in which primary specimens obtained from patients undergoing surgical resection were collected fresh either from the primary tumor or from metastatic lymph nodes. The specimen was immersed in saline solution and kept refrigerated. It was implanted within 24 hours under the skin of Rag2/cytokine receptor common $\gamma$-chain double knockout (Rag2 $\gamma$ DKO) mice, either as small ( $<2 \mathrm{~mm}$ ) fragments of tumor or as cell suspensions in 50\% matrigel (Becton Dickinson; Franklin Lakes, NJ), ranging from 1 to 5 million total cells per injection. There was no difference in success rate when implanting as single cell digests or small, nondigested tumor pieces. When the surgical specimen was sufficiently large and there was enough tissue for analysis, flow cytometry was performed from the human tumor after dissociation into single cells. The singlecell suspensions were washed in Hank's balanced salt solution (HBSS)/2\% heat inactivated calf serum (HICS) and counted and then resuspended in $100 \mu \mathrm{L}$ per $10^{6}$ cells of HBSS and incubated with $1 \mathrm{mg} / \mathrm{mL}$ mouse IgG (Sigma, St. Louis, MO) for 10 minutes. The suspensions were then washed with HBSS/2\% HICS, resuspended in $100 \mu \mathrm{L}$ per $10^{6}$ cells of HBSS, and stained with antibodies. Anti-CD44 (Pharred, phycoerythrin-, or allophycocyanin-conjugated, clone G44-26; BD Pharmingen) was used at a 1:50 dilution; lineage markers anti-CD2, CD3, CD10, CD16, CD18, CD31, CD64, and CD140b (all diluted 1:50; BD Pharmingen, Franklin Lakes, NJ) were used to allow identification of contaminating nontumor cells from patient samples. In each tumor analyzed by flow cytometry, a distinct population of Lin-CD44+ and Lin-CD44- cancer cells was identifiable, and the percentage of Lin-CD44+ cells was recorded (Figure 1).

Clinical and pathologic data were collected, including age, sex, alcohol and tobacco consumption, site of tumor, TNM stage, prior treatment, and current planned treatment (surgery/radiotherapy/chemo). Histologic criteria were reviewed by 1 pathologist (R.P.) and included grade, extracapsular spread, perineural invasion, angiolymphatic invasion, and margins of resection. Human papillomavirus status was not recorded because only 4 tumors were from the oropharynx. Patients were followed up every 4 to 6 weeks for the first year after diagnosis and every 8 to 12 weeks thereafter. If the patient did not appear for follow-up, his primary care physician or referring 


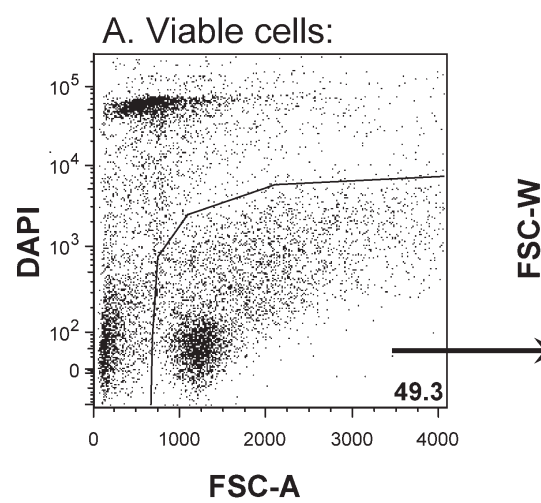

B. Singlets:

C. Lin-:
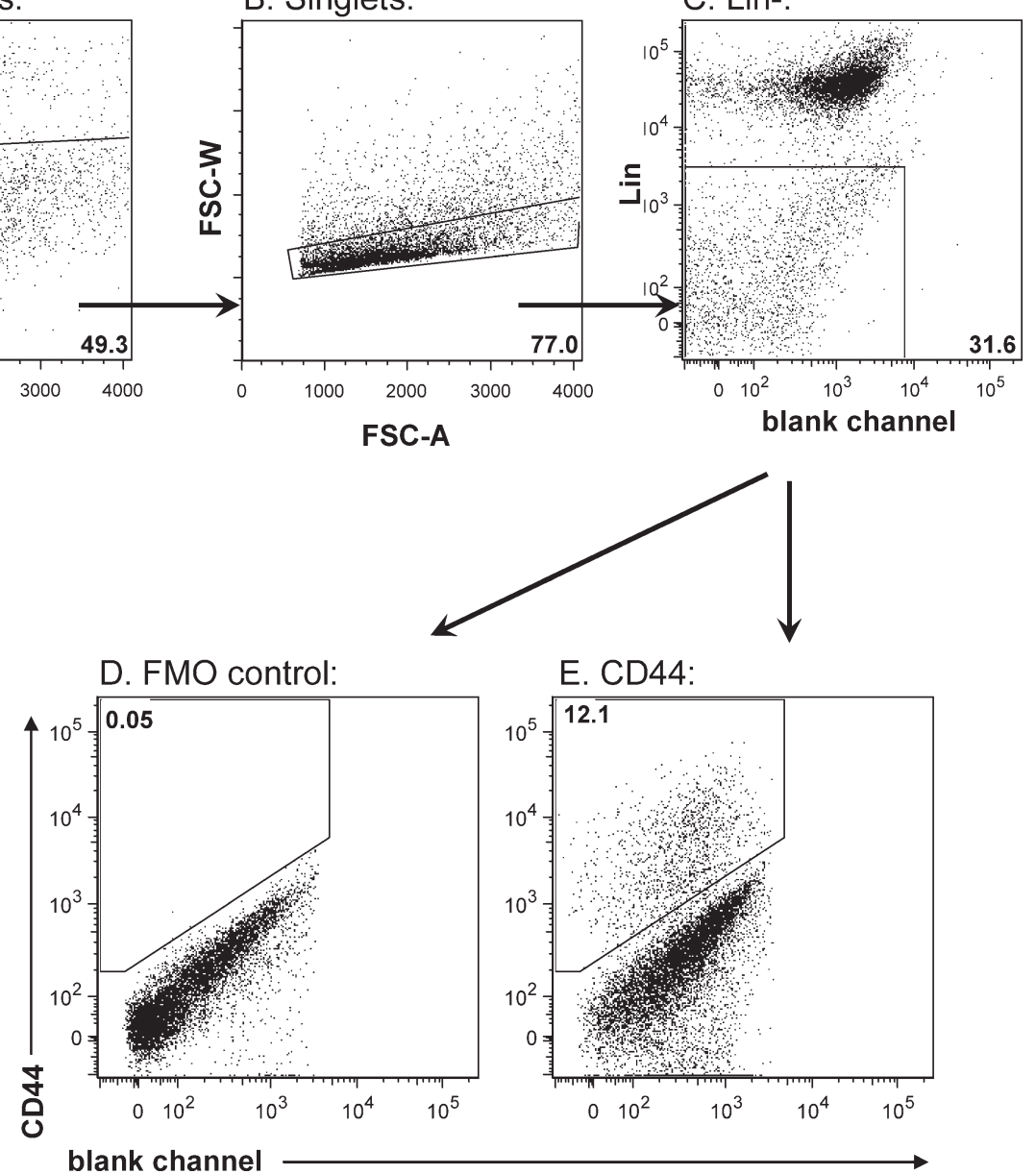

FIGURE 1. Flow cytometric analysis of a typical HNSCC sample. Cells are sequentially gated on (A) viable cells with either DAPI or propidium iodide as a dead cell marker, then (B) doublets are excluded in a forward scatter area versus width profile. The lineage negative cells are then excluded (C), and finally, the gate for CD44+ cells is drawn by use of a "fluorescence-minus-one" control, in which the CD44 antibody is excluded from the staining mixture (D). When this gate is applied to the CD44 stained sample, the frequency of Lin-CD44+ cells is determined (E). In this case the frequency of Lin-CD44+ cells is $12.1 \%$.

otolaryngologist-head and neck surgeon was contacted. Outcome was evaluated for site of failure (local, regional or distant) and overall and disease-specific survival.

Statistical Analysis. Results were evaluated for normality. Correlations between continuous variables were done with the Pearson or the Spearman coefficients of correlation, for parametric or nonparametric groups, respectively. The best cut-off points for discriminating between good and bad prognostic groups were determined after applying receiver operating characteristic analysis. The best cut-off point was determined by (sensitivity - [1-specificity]), and sensitivity was favored. Univariate analysis of survival for the measured variables was performed by constructing Kaplan Meier curves, and statistical significance was tested by use of the log-rank test. Cox proportional hazard model to evaluate parameters that influence the survival of the patients was applied to test which of the parameters found on a univariate analysis was independent.

\section{RESULTS}

The following are examples of 4 patients, including a brief history, treatment follow-up, and percentage of Lin-CD44+ cells from the tumor specimen. In these selected cases it can be appreciated that the patients who did poorly also had a higher Lin-CD44+ frequency in their tumors.

Patient A, a 52-year-old man, was initially seen with a T2N2b (T/N/M classification) squamous cell carcinoma (SCC) of the left lateral tongue. He was treated in July 2004 by partial glossectomy and modified radical neck dissection. Pathologic study showed moderately differentiated SCC, margins clear of tumor, and 2/62 positive lymph nodes, with no perineural invasion or extracapsular invasion. The patient underwent postoperative radiation. He was last seen on July 2009 without evidence of disease. The Lin- 


\begin{tabular}{lc}
\hline \multicolumn{2}{c}{ Table 1. Summary of parameters analyzed. } \\
\hline Parameter & No. of patients $(\%)$ \\
\hline Site & \\
Oral cavity & $13(42 \%)$ \\
Skin or lip & $8(26 \%)$ \\
Oropharynx & $4(13 \%)$ \\
Larynx and hypopharynx & $4(13 \%)$ \\
Nose and sinuses & $2(6 \%)$ \\
T classification & \\
T0 & $3(10 \%)$ \\
T1 & $1(3 \%)$ \\
T2 & $6(19 \%)$ \\
T3 & $7(22 \%)$ \\
T4 & $14(45 \%)$ \\
N classification & \\
N0 & $13(42 \%)$ \\
N1 & $0(0 \%)$ \\
N2 & $11(35 \%)$ \\
N3 & $7(23 \%)$ \\
Alcohol & \\
$>3$ drinks/day & $8(26 \%)$ \\
1-3 drinks/day & $10(32 \%)$ \\
$<1$ drink/day & $13(42 \%)$ \\
Tobacco & \\
$>25$ pack year & $13(42 \%)$ \\
1-25 pack year & $6(19 \%)$ \\
$<1$ pack year & $12(39 \%)$ \\
Lin-CD44+ cell frequency $(n=22)$ & \\
Mean \pm SD & $25 \%(0.4 \%-80.6 \%)$ \\
Median & $17 \%$ \\
Successful xenograft $(n=30)$ & \\
Yes & $16(53 \%)$ \\
No & $14(47 \%)$ \\
\hline &
\end{tabular}

CD44+ was $1.8 \%$, and the tumor did not xenotransplant into mice.

Patient B, a 54-year-old man, presented with a T2N0M0 (T/N/M classification) SCC of the right mobile tongue. He was treated in October 2006 by partial glossectomy. The pathologic study showed moderately differentiated SCC with perineural invasion. After surgery the patient underwent radiation to the tongue and neck. On March 2007 a short interval recurrence in the right neck was noted for which a modified radical neck dissection was performed. Pathologic examination revealed 1 positive node $2.5 \mathrm{~cm}$ with extracapsular extension. As of January 2010 the patient is free of disease. The Lin-CD44+ from the first surgery was $23 \%$, and the tumor was xenografted successfully.

Patient C, a 56-year-old woman, was previously treated by chemotherapy and radiation for floor of mouth SCC. There was recurrence in the floor of mouth, T/N/M classification T4aN2bM0. The patient was treated by a composite resection and a modified radical neck dissection and reconstruction with a fibula free flap. Pathologic examination showed complete resection with clear margins and no perineural invasion or angiolymphatic invasion. In July 2007 local recurrence was noted, and the patient underwent palliative chemotherapy. She died in January 2008. The Lin-CD44+ from the surgery (2007) was 78\%, and the tumor was xenografted successfully.
Patient D, a 35-year-old man, was diagnosed with a T1N0M0 (T/N/M classification) left lateral tongue SCC that was treated in June 2005 by partial glossectomy and left supraomohyoid neck dissection. Margins were not involved, and there were no positive lymph nodes. In January 2006 a recurrence was noted in the neck and tongue (rT2N3M0). The patient was treated surgically with a partial glossectomy, partial pharyngotomy, and left radical neck dissection. The postoperative course was uneventful. During urgent radiation planning, a neck recurrence was noted. The patient died in May 2006. The Lin-CD44+ frequency from the second surgery was $72.8 \%$, and the tumor was xenotransplanted successfully.

The study included 31 patients with HNSCC, with a mean age of $61.5 \pm 2.8$ years (mean \pm SEM) and a mean follow-up of $12.9 \pm 1.14$ months (mean \pm SEM), range, 4.9-27 months. Twenty-four patients were male, and seven patients were female. Out of this group, 21 patients (68\%) were newly diagnosed, whereas 10 patients were treated because of recurrent disease. The primary sites and TNM stages are shown in Table 1.

All patients had SCC: 4 were well differentiated (13\%), 19 were moderately differentiated $(61 \%)$, and 8 were poorly differentiated (26\%). Other pathologic parameters, such as perineural invasion, extracapsular spread, and angiolymphatic invasion, are shown in Table 2.

Subsequent failure occurred in 15 patients (48\%): local failure in $6(19 \%)$, regional failure in $8(26 \%)$, and distant failure in $4(13 \%)$. At the end of the study, $22 / 31$ patients $(71 \%)$ were alive.

CD44 was examined in 22 patients, with a mean Lin-CD $44+$ cell frequency of $25.4 \% \pm 5.4 \%$ (SEM), median $17 \%$, (range $0.4-80.6 \%$ ). Successful xenograft implantation in Rag $\gamma \mathrm{DKO}$ mice was obtained for 16 tumors of $30(53 \%)$.

Correlation of Frequency of Lin-CD44+ Cells to Pathologic Parameters. There was a trend toward an inverse correlation between tumor grade and mean Lin-CD44+ cell frequency: poorly differentiated carcinoma $45.8 \%$, moderately differentiated carcinoma $21 \%$, and well-differentiated carcinoma $11.8 \%$ ( $p$ $=.09$; Table 2). A higher frequency of Lin-CD44+ cells was found in patients with perineural invasion, as well as angiolymphatic invasion, but these were not found to be statistically significant. There was no correlation of extracapsular spread to Lin-CD44+ cell frequency.

Correlation of Frequency of Lin-CD44+ Cells to Clinical Parameters. A significant correlation was found between T classification and Lin-CD44+ cell frequency, $p=.05$ : patients with T0-2 disease ( 7 patients) had a mean frequency of $10.3 \%$ compared with T3-4 (15 patients) with a mean frequency of $32.4 \%$. There 


\begin{tabular}{|c|c|c|c|c|}
\hline Parameter & No. of patients & Lin-CD44+ \% (Mean \pm SEM) & No. of patients & Successful implantation \\
\hline T classification & & $p=.05$ & & NS \\
\hline Т 0-2 & 7 & $10.3 \pm 3.5$ & 9 & $5(56 \%)$ \\
\hline Т 3-4 & 15 & $32.4 \pm 7.1$ & 21 & $11(52 \%)$ \\
\hline $\mathrm{N}$ classification & & NS & & NS \\
\hline NO & 10 & $23.98 \pm 6.01$ & 13 & $7(54 \%)$ \\
\hline N2 & 6 & $21.43 \pm 12.2$ & 10 & $5(50 \%)$ \\
\hline N3 & 6 & $31.58 \pm 13.2$ & 7 & $4(57 \%)$ \\
\hline Site & & NS & & NS \\
\hline Skin and lip & 8 & $26.77 \pm 8.4$ & 8 & $4(50 \%)$ \\
\hline Oral cavity & 13 & $26.8 \pm .12 .2$ & 12 & $8(67 \%)$ \\
\hline Oropharynx and nasopharynx & 4 & $11.07 \pm 3.7$ & 4 & $1(25 \%)$ \\
\hline Larynx and hypopharynx & 4 & $32.77 \pm 24.4$ & 4 & $1(25 \%)$ \\
\hline Nose and paranasal sinuses & 2 & $33.45 \pm 1.65$ & 2 & $2(100 \%)$ \\
\hline Failure & & $p=.05$ & & $p=.009$ \\
\hline With failure & 11 & $35.65 \pm 7.3$ & 15 & $12(80 \%)$ \\
\hline Local failure & & $p=.03$ & & NS \\
\hline No local failure & 16 & $18.66 \pm 5.9$ & 24 & $12(50 \%)$ \\
\hline With local failure & 6 & $43.20 \pm 8.5$ & 6 & $4(67 \%)$ \\
\hline Regional failure & & NS & & $p=.003$ \\
\hline No regional failure & 17 & $21.84 \pm 5.4$ & 22 & $8(36 \%)$ \\
\hline With regional failure & 5 & $37.32 \pm 14.9$ & 8 & $8(100 \%)$ \\
\hline Status & & $p=.11$ & & NS \\
\hline Alive & 14 & $18.95 \pm 5.8$ & 21 & $11(56 \%)$ \\
\hline Dead & 8 & $36.58 \pm 10$ & 9 & $5(52 \%)$ \\
\hline Previous treatment & & $p=.11$ & & NS \\
\hline Previous treatment & 9 & $35.6 \pm 10.2$ & 10 & $5(50 \%)$ \\
\hline No previous treatment & 13 & $18.2 \pm 5.22$ & 20 & $9(45 \%)$ \\
\hline Grade & & $p=.09$ & & $p=.015$ \\
\hline Well differentiated & 3 & $11.80 \pm 1.8$ & 4 & $0(0 \%)$ \\
\hline Moderately differentiated & 14 & $20.96 \pm 6.5$ & 18 & $9(50 \%)$ \\
\hline No & 7 & $13.31 \pm 5.9$ & 12 & $4(33 \%)$ \\
\hline Extracapsular extension & & NS & & NS \\
\hline Yes & 8 & $29.0 \pm 10.7$ & 9 & $5(56 \%)$ \\
\hline No & 4 & $23.25 \pm 16.9$ & 7 & $4(57 \%)$ \\
\hline Angiolymphatic invasion & & $p=.23$ & & NS \\
\hline Yes & 6 & $35.88 \pm 13.7$ & 8 & $5(63 \%)$ \\
\hline No & 16 & $21.41 \pm 5.3$ & 22 & $11(50 \%)$ \\
\hline Successful implantation & & $p=.009$ & & \\
\hline No & 11 & $13.52 \pm 3.7$ & & \\
\hline Yes & 10 & $40.81 \pm 9$ & & \\
\hline
\end{tabular}

Abbreviation: NS, not significant.

was no correlation of Lin-CD44+ cell frequency to $\mathrm{N}$ classification, nor to overall stage. No difference was found in Lin-CD44+ cell frequency among the different sites of head and neck carcinomas. There was a trend toward increased frequency in patients who were treated because of recurrent disease compared with patients who had primary diagnosed tumors ( $35.7 \%$ vs $18.3 \%$, respectively), but it was not significant $(p=.1119)$.

The Lin-CD44+ cell frequency was significantly higher in patients with subsequent failure, compared with patients without failure: $35.7 \%$ vs $15 \%(p=.05)$. Significantly higher frequencies of Lin-CD44+ cells were found in patients with local failure $(43.2 \%)$ compared with patients without local failure $(18.7 \% ; p=$ .038, Table 2). Multivariate analysis showed that the fraction of Lin-CD44+ was significantly correlated to failure $(p=.02)$.

An elevated frequency of Lin-CD44+ cells was also found in patients with regional failure and distant failure; however, it was not significant. The best cut-off point for predicting overall failure was detected after applying receiver operating characteristic analysis. The best cut-off point of Lin-CD44+ cell frequency for discriminating between good and bad prognostic groups was $15.2 \%$. Sensitivity: 0.91 , specificity: 0.73 , [sensitivity $-(1$-specificity) $]=0.64$, area under curve $=0.818$ ). A Kaplan Meier curve for overall failure demonstrates a significantly lower incidence of failure in patients with a Lin-CD44+ cell frequency $<15 \%$ compared with patients with a Lin-CD44+ cell frequency $>15 \%$ ( $p=$ .005 , log-rank test; Figure 2). 


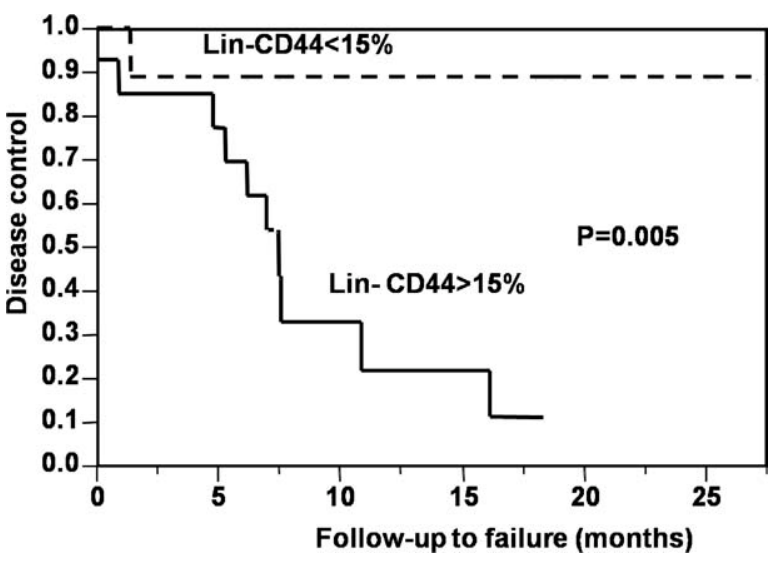

FIGURE 2. Kaplan Maier analysis of disease control stratified by Lin-CD44+ cell frequency $<15 \%(n=10)$ versus $>15 \%(n=12)$.

A Kaplan Meier curve for overall survival showed significant differences as well. Patients with a LinCD44+ cell frequency $<15 \%$ had an $88 \%$ overall survival rate at 27 months, compared with $30 \%$ at 18 months for patients with a Lin-CD44+ cell frequency $>15 \%(p=.01, \log$ rank test; Figure 3$)$.

CD44 and Xenograft Implantation. A significant correlation was found between the frequency of LinCD44+ cells and successful xenograft tumor implantation into Rag $\gamma$ DKO mice: the mean frequency of Lin-CD44+ cells was $40.8 \%$ for successful xenograft implantation, compared with $13.5 \%$ for those with failed xenograft implantation $(p=.009)$.

\section{Correlation of Xenograft Implantation to Pathologic}

Parameters. Successful xenograft implantation was significantly correlated to tumor grade $(p=0.015)$ : none of the 4 well-differentiated tumors were successfully xenografted, compared with $50 \%$ (9 of 18) of the moderately differentiated tumors and $88 \%$ ( 7 of 8 ) of the poorly differentiated tumors. Higher xenograft rates were found for tumors with perineural invasion (67\%) compared with

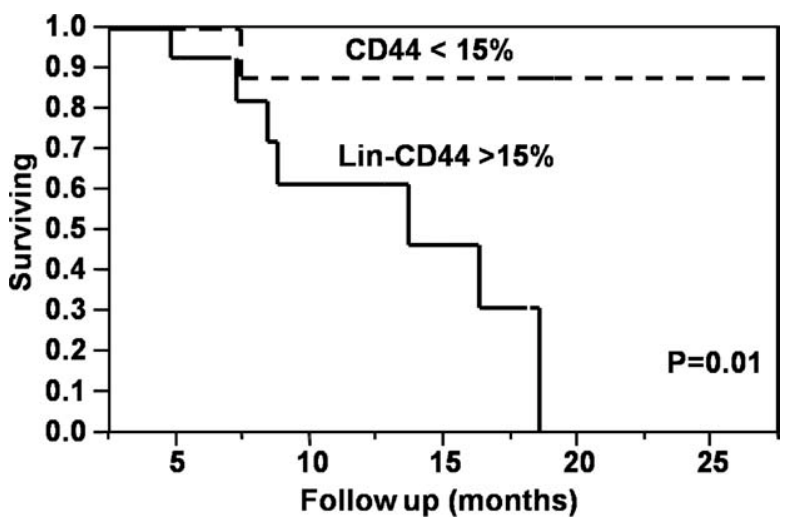

FIGURE 3. Kaplan Meier survival analysis stratified by LinCD $44+$ cell frequency $<15 \%(n=10)$ versus $>15 \%(n=12)$.

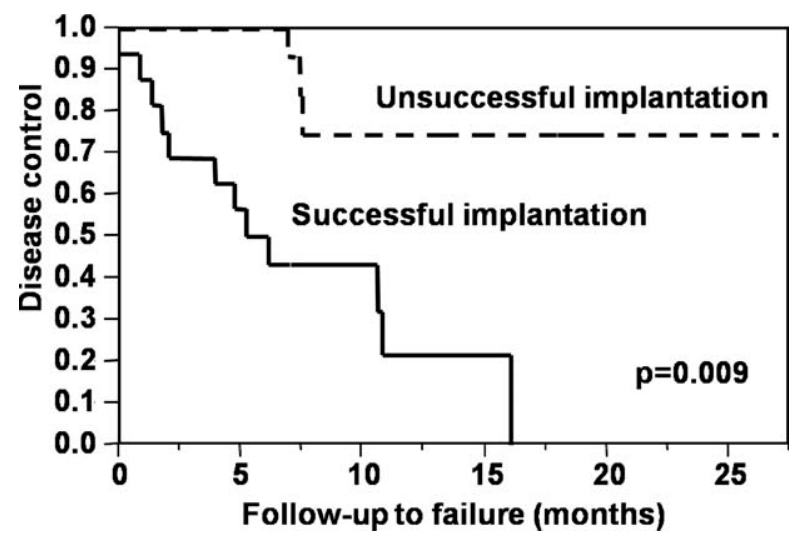

FIGURE 4. Kaplan Maier Analysis of disease control stratified by successful tumor implantation $(n=16)$ versus unsuccessful xenograft implantation $(n=14)$ in RaggDKO mice.

those without perineural invasion (33\%); however, it was not significant (Fisher exact test $p=.13$ ).

Xenograft Implantation: Correlation to Clinical Parameters. There was no correlation between successful xenograft implantation and $\mathrm{T}$ classification, $\mathrm{N}$ classification, overall stage, or site of tumor. There was no difference in the percent of successful implantation between patients who were newly diagnosed ( 9 successful implantations of 20 [45\%]) compared with patients who had previous treatment (5 successful implantations of 10 [50\%]).

There was a trend toward increased successful implantation in patients who do not smoke, compared with smokers: 9 of $12(75 \%)$ non-smokers had successful implantation, compared with 7 of $18(39 \%)$ of smokers $\left(\chi^{2}, p=.05\right.$, Fisher exact test $\left.p=.07\right)$. Successful implantation was most significantly correlated to subsequent failure: 12 of 15 patients (80\%) who failed had successful implantation, compared with 4 of $11(27 \%)$ of the patients who did not fail (Fisher exact test $p=.009$ ).

No significant correlation was found between successful implantation and local failure, but there was a significant correlation to regional failure (Fisher exact test $p=.003)$ : all patients with regional failure (8 patients, 100\%) had successful implantation, compared to $36 \%$ (8 of 22) of the patients without regional failure. A Kaplan Meier curve was generated for overall failure, stratified by successful implantation. A significant difference was found in failure rate between patients with successful implantation compared to those without implantation ( $p=.009$, log-rank test; Figure 4$)$. There was no significant difference in the survival rate of patients whose tumor had successful implantation compared with those whose tumor had not.

In Cox proportional hazard model to evaluate parameters that influence the survival of the patients, including Lin-CD44+ and successful tumor implantation, Lin-CD44+ was a significant and independent 
parameter that influenced survival, whereas successful implantation was not significant $(p=.049)$.

\section{DISCUSSION}

CD44 proteins are a widely expressed family of adhesion molecules encoded by a single highly conserved gene. The different family members are generated by posttranslational modifications and extensive alternative splicing. ${ }^{24}$ The standard CD44 isoform (CD44s) contains 10 of the 19 exons and is widely expressed. The remaining exons are alternatively spliced and incorporated into the standard backbone to create several variant isoforms, which are expressed in a variety of tissues at particular periods in development, or in cancer cells. Previous studies have shown conflicting results regarding the significance of CD44 expression in head and neck tumors. ${ }^{25-35}$ Most studies found that using immunohistochemistry, underexpression of CD44, or one of the variants is a predictor of reduced survival. In contrast, others have found that CD44 is consistently highly expressed on all head and neck tissues, from normal to HNSCC. ${ }^{36}$ These varying results are likely due to the use of different antibodies by different investigators, as well as different methods of tissue processing, staining, and enumeration of results. Given the complexity of the molecule, the variation in results already present in the literature, and the fact that our analysis was done by an entirely different method (ie, flow cytometry), it is not surprising that there are conflicts with the published data. Some possible explanations for these conflicts are likely due to two major differences between our study and those previously described: the use of a pan-CD44 antibody, and the use of flow cytometry. Many previous studies used antibodies for specific variants of CD44. Although the expression of specific variants may be reduced in tumors with a worse prognosis, the overall CD44 expression may not. It is of interest for future studies to determine which variants of CD44 are expressed in the tumorinitiating subset of head and neck cancer cells versus normal oral squamous epithelial stem cells and how differences in their expression patterns affect the behavior of these cells. The use of flow cytometry is a highly quantitative method, compared with previous studies that analyzed CD44 with immunohistochemistry and a subjective examiner-based analysis reporting positive or negative results on a semiquantitative scale (for example, by assigning scores of $-/+/++/$ +++ ). CD44 is also highly expressed on stromal and inflammatory cells, which we exclude when we perform flow cytometric analysis (that is the Lin- component of the analysis). Although it is possible on histologic study to differentiate between tumor and nontumor cells, it may not be as accurate at excluding these. Finally, immunohistochemistry analyzes a significantly smaller number of cells and looks at only small regions of the tumor; on the other hand, a large piece of tumor is dissociated for flow cytometry and hundreds of thousands to millions of cells are run through the machine, allowing us to typically acquire at least 100,000 viable cell events for analysis. Because the tumor was dissociated, the value we get is an average across the whole tissue sample rather than one thin section.

On the basis of previous studies showing that the Lin-CD44+ fraction of HNSCC cells contains a cancer stem cell population, our study suggests that a higher frequency of cancer stem cells is associated with a worse prognosis. This would support the theory that stem cell burden and not overall tumor burden is the important prognostic finding. This hypothesis is supported by a recent article demonstrating the importance of the stem cell signature in predicting prognosis in breast cancer, ${ }^{19}$ where tumors with a genetic signature similar to the breast cancer stem cells had a worse prognosis. An alternative explanation not relevant to a stem cell hypothesis is that the CD44 protein is merely a marker for aggressiveness of the tumor, and that CD44 plays a functional role in tumor progression by mediating, for example, homing or binding at sites of metastases. However, in support of our hypothesis, it has also recently been shown that aldehyde dehydrogenase $(\mathrm{ALDH})$ activity can be used as a HNSCC stem cell marker, with ALDH+ cells representing $1 \%$ to $7.8 \%$ of tumor cells, and xenografts initiated from as few as 500 cells. ${ }^{37,38}$ Chen et $\mathrm{al}^{38}$ also showed that high ALDH1 protein levels were positively associated with stage and grade of HNSCC and with poor outcome. To determine whether cancer stem cell frequency is truly a determinant of patient outcome will require additional studies with limiting dilution assays to functionally measure the cancer stem cell frequencies, as well as the identification of additional markers to identify more precisely the cancer stem cell population, as has been done in other cancers such as breast cancer and leukemias.

This study suggests that successful tumor xenograft in mice was also an important prognostic sign. The frequency of Lin-CD44+ cells correlated with successful implantation and therefore may explain this finding. However, it seems there are other factors as well, because the frequency of Lin-CD44+ cells correlated better with local recurrence whereas success of implant correlated better with regional recurrence (Table 2). In addition, tumor grade correlated better with successful xenografting than with frequency of Lin-CD44+ cells (Table 2). This implies that the success of xenograft implants is related to other parameters, such as ability to survive in a foreign microenvironment, in addition to the frequency of Lin-CD44+ cells in HNSCC.

Limitations and Future Directions. It should be stressed that the findings in this study, although significant, are somewhat preliminary because only 31 
patients were recruited and the tumors were very diverse, including nasal, skin, oral cavity, oropharynx, and laryngohypopharynx. In addition we combined new cases with recurrences. Because of the limited number of patients, a meaningful multivariate analysis could not be done. As we and others gather more patients to the database, these findings can be further tested.

Sorting for Lin-CD44+ cells enriches the stem cell population; however, to test the theory further the population must be more refined. The percentage of actual stem cells is likely much lower than the frequency of Lin-CD44+ cells reported in this study.

\section{CONCLUSIONS}

This study suggests that a flow cytometric measurement of the frequency of Lin-CD44+ cells may provide a prognostic test for patients with HNSCC. In addition and arguably more importantly, it lends preliminary support to the notion that to better understand HNSCC tumor behavior, research should be focused on the understanding of cancer stem cells, the earliest steps of their dysregulation, and the development of methods for their eradication.

\section{REFERENCES}

1. Jain S, Khuri FR, Shin DM. Prevention of head and neck cancer: current status and future prospects. Curr Probl Cancer 2004;28:265-286.

2. Lee JT, Ko CY. Has survival improved for nasopharyngeal carcinoma in the United States? Otolaryngol Head Neck Surg 2005; 132:303-308

3. Golas SM. Trends in palatine tonsillar cancer incidence and mortality rates in the United States. Community Dent Oral Epidemiol 2007;35:98-108.

4. Gupta S, Kong W, Peng Y, Miao Q, Mackillop WJ. Temporal trends in the incidence and survival of cancers of the upper aerodigestive tract in Ontario and the United States. Int J Cancer 2009;125:2159-2165.

5. Pardal R, Clarke MF, Morrison SJ. Applying the principles of stem-cell biology to cancer. Nat Rev Cancer 2003;3:895-902.

6. Reya T, Morrison SJ, Clarke MF, Weissman IL. Stem cells, cancer, and cancer stem cells. Nature 2001;414(6859):105-111.

7. Blair A, Hogge DE, Ailles LE, Lansdorp PM, Sutherland HJ. Lack of expression of Thy-1 (CD90) on acute myeloid leukemia cells with long-term proliferative ability in vitro and in vivo. Blood 1997;89:3104-3112.

8. Bonnet D, Dick JE. Human acute myeloid leukemia is organized as a hierarchy that originates from a primitive hematopoietic cell. Nat Med 1997;3:730-737.

9. Jamieson CH, Ailles LE, Dylla SJ, et al. Granulocyte-macrophage progenitors as candidate leukemic stem cells in blast-crisis CML. N Engl J Med 2004;351:657-667.

10. Lapidot T, Sirard C, Vormoor J, et al. A cell initiating human acute myeloid leukaemia after transplantation into SCID mice. Nature 1994;367(6464):645-648.

11. Miyamoto T, Weissman IL, Akashi K. AML1/ETO-expressing nonleukemic stem cells in acute myelogenous leukemia with 8;21 chromosomal translocation. Proc Natl Acad Sci U S A 2000;97:7521-7526.

12. Ailles LE, Weissman IL. Cancer stem cells in solid tumors. Curr Opin Biotechnol 2007;18:460-466.

13. Rosen JM, Jordan CT. The increasing complexity of the cancer stem cell paradigm. Science 2009;324(5935):1670-1673.

14. Quintana E, Shackleton M, Sabel MS, Fullen DR, Johnson TM, Morrison SJ. Efficient tumour formation by single human melanoma cells. Nature 2008;456(7222):593-598.
15. Shackleton M, Quintana E, Fearon ER, Morrison SJ. Heterogeneity in cancer: cancer stem cells versus clonal evolution. Cell 2009; 138:822-829.

16. Gupta PB, Chaffer CL, Weinberg RA. Cancer stem cells: mirage or reality? Nat Med 2009;15:1010-1012.

17. Robbins KT, Doweck I, Samant S, Vieira F, Kumar P. Factors predictive of local disease control after intraarterial concomitant chemoradiation (RADPLAT). Laryngoscope 2004;114:411-417.

18. Massano J, Regateiro FS, Januario G, Ferreira A. Oral squamous cell carcinoma: review of prognostic and predictive factors. Oral Surg Oral Med Oral Pathol Oral Radiol Endod 2006;102:67-76.

19. Liu R, Wang X, Chen GY, et al. The prognostic role of a gene signature from tumorigenic breast-cancer cells. N Engl J Med 2007;356:217-226.

20. Prince ME, Sivanandan R, Kaczorowski A, et al. Identification of a subpopulation of cells with cancer stem cell properties in head and neck squamous cell carcinoma. Proc Natl Acad Sci U S A 2007;104:973-978.

21. Ailles L, Prince M. Cancer stem cells in head and neck squamous cell carcinoma. Methods Mol Biol 2009; 568: 175-193.

22. Locke M, Heywood M, Fawell S, Mackenzie IC. Retention of intrinsic stem cell hierarchies in carcinoma-derived cell lines. Cancer Res 2005;65:8944-8950.

23. Nelson AD, Grandis JR. The role of CD44 in HNSCC. Cancer Biol Ther 2007;6:125-126.

24. Ponta H, Sherman L, Herrlich PA. CD44: from adhesion molecules to signalling regulators. Nat Rev Mol Cell Biol 2003;4:3345 .

25. Bankfalvi A, Krassort M, Buchwalow IB, Vegh A, Felszeghy E, Piffko J. Gains and losses of adhesion molecules (CD44, E-cadherin, and beta-catenin) during oral carcinogenesis and tumour progression. J Pathol 2002; 198:343-351.

26. Carinci F, Stabellini G, Calvitti M, et al. CD44 as prognostic factor in oral and oropharyngeal squamous cell carcinoma. J Craniofac Surg 2002;13:85-89.

27. Gonzalez-Moles MA, Bravo M, Ruiz-Avila I, et al. Adhesion molecule CD44 as a prognostic factor in tongue cancer. Anticancer Res 2003;23(6D):5197-202.

28. Gonzalez-Moles MA, Gil-Montoya JA, Ruiz-Avila I, Esteban F, Delgado-Rodriguez M, Bascones-Martinez A. Prognostic significance of p21WAF1/CIP1, p16INK4a and CD44s in tongue cancer. Oncol Rep 2007;18:389-396.

29. Kosunen A, Pirinen R, Ropponen K, et al. CD44 expression and its relationship with MMP-9, clinicopathological factors and survival in oral squamous cell carcinoma. Oral Oncol 2007;43:51-59.

30. Kuo MY, Cheng SJ, Chen HM, Kok SH, Hahn LJ, Chiang CP. Expression of CD44s, CD44v5, CD44v6 and CD44v7-8 in betel quid chewing-associated oral premalignant lesions and squamous cell carcinomas in Taiwan.J Oral Pathol Med 1998;27:428-433.

31. Masuda M, Kuratomi Y, Shiratsuchi H, Nakashima T, Naonobu $\mathrm{K}$, Komiyama S. Decreased CD44H expression in early-stage tongue carcinoma associates with late nodal metastases following interstitial brachytherapy. Head Neck 2000;22:662-665.

32. Qin G, Li W, Sun X, Zhu L, Chen Z. [Expression and significance of CD44v6 and MMP-9 in laryngeal squamous cell carcinoma]. Lin Chuang Er Bi Yan Hou Ke Za Zhi 2005;19:688-691.

33. Sato S, Miyauchi M, Takekoshi T, et al. Reduced expression of CD44 variant 9 is related to lymph node metastasis and poor survival in squamous cell carcinoma of tongue. Oral Oncol 2000;36:545-549.

34. Stoll C, Baretton G, Soost F, Terpe HJ, Domide P, Lohrs U. Prognostic importance of the expression of CD44 splice variants in oral squamous cell carcinomas. Oral Oncol 1999;35:484-489.

35. Wang SJ, Wong G, de Heer AM, Xia W, Bourguignon LY. CD44 variant isoforms in head and neck squamous cell carcinoma progression. Laryngoscope 2009;119:1518-1530.

36. Mack B, Gires O. CD44s and CD44v6 expression in head and neck epithelia. PLoS One 2008;3:e3360.

37. Clay MR, Tabor M, Owen JH, et al. Single-marker identification of head and neck squamous cell carcinoma cancer stem cells with aldehyde dehydrogenase. Head Neck 2010;32:1195-1201.

38. Chen YC, Chen YW, Hsu HS, et al. Aldehyde dehydrogenase 1 is a putative marker for cancer stem cells in head and neck squamous cancer. Biochem Biophys Res Commun 2009;385:307-313. 